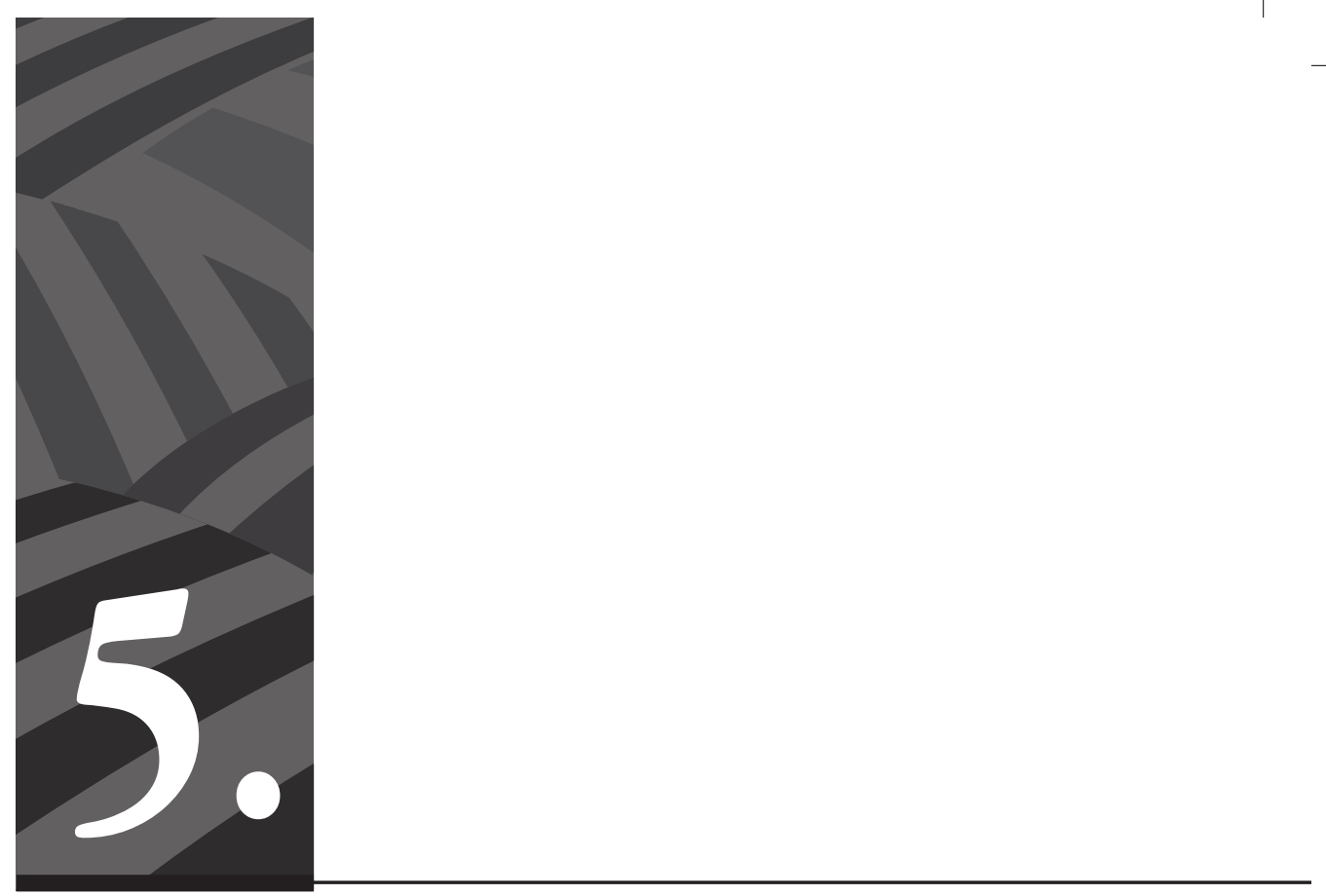

La paz difícil. El reconocimiento de la historia y los actores de paz 


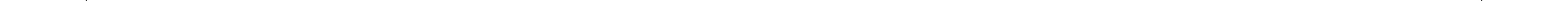




\title{
La paz difícil. El reconocimiento de la historia y los actores de paz
}

\author{
Por Hernán Rodríguez Vargas*
}

A mis amigos de Ágape**, como muestra de mi afecto y compromiso.

Necesitamos la historia, pero la necesitamos no como el holgazán malcriado en los jardines del saber ${ }^{* * *}$

NIETZSCHE

Resumen: A la pregunta por recorrer, ipara quién es la paz?, una respuesta, diríamos, desalentadora: para los vencidos. Más que desalentadora, realista, en un sentido radical, es decir, negativa y positivamente. Este trabajo busca examinar quiénes son los vencidos y cómo son las múltiples formas en las que hacen, construyen y elaboran procesos de paz —incluso, es posible examinar contra quiénes- . No solo la guerra tiene antagonismos, hacer la paz también los tiene. De estos reconocimientos podremos hacer el tránsito de "vencidos" a actores de paz, que, a su vez, han de asumirse, y se asumen, en lo que aquí denominamos la paz difícil, concepto a desarrollar a lo largo del trabajo, y desde el cual se evidencian de forma crítica algunos retos y desafíos que implican aproximarnos a nuevas formas de comprendernos socialmente, una vez se dejan atrás las opciones violentas para tramitar conflictos.

Palabras clave: paz difícil, vencidos, actores de paz, conflicto.

* Docente investigador colombiano. Profesional en Estudios Literarios, filósofo y magíster en Historia de la Pontificia Universidad Javeriana.

** Aunque este texto no profundiza en la labor de esta fundación, en buena medida está inspirado en las personas que allí trabajan, quienes me han enseñado, como actores de paz, a creer en la acción política que ella implica, la consecución y el buen término de este sueño común. Para el lector interesado, puede ver su página web: http://www.agapeforcolombia.com/?lang = es

*** Cita que aparece también en la tesis XII de Benjamín, Walter (2005). Tesis sobre la historia y otros fragmentos. 


\section{The difficult peace. On self-recognition of history and peace actors}

Abstract: A discouraging answer, we would say, to the question "for whom is peace?" would be: for the vanquished. More than discouraging, realistic in a radical sense, that is, negatively and positively. This work seeks to examine who are the losers and how are the multiple ways in which they make, build and elaborate peace processes; it is even possible to examine against whom. Not only does war have antagonisms, making peace has them too. From these acknowledgments we can make the transition from "losers" to actors of peace, which, in turn, have to be assumed, as they assume themselves, as what we here call the difficult peace; a concept to develop throughout the work, and from which are critically evidenced some challenges that involve approaching new ways of social self-understanding once we have left behind the violent options to process a multiplicity of conflicts, in present, past and future.

Keywords: Difficult peace, vanquished, peace actors, conflict.

Cómo citar este artículo: Rodríguez Vargas, Hernán (2018). La paz difícil. El reconocimiento de la historia y los actores de paz. Revista Controversia, 211, 165-181.

Fecha de recepción: 27 de junio de 2018

Fecha de aprobación: 10 de septiembre de 2018

\section{Introducción}

$\mathrm{E}$

n Colombia, en el marco de la coyuntura política actual en la que se insiste desde distintos lugares de enunciación en hablar de paz, aparecen dos preguntas por responder: ¿qué es la paz? y ¿cómo se construye paz? A cambio, y para responder estas dos, quisiera formular la pregunta: ipara quién es la paz? Y quisiera, además, ponerlo todo en plural: ¿para quiénes son las paces? Esto, en la medida en que comprendo que, a pesar de que la idea de paz puede llegar a funcionar, entre encuentros y desencuentros, como un motor que moviliza todo tipo de acciones, variadas y diversas, en contextos específicos se configuran no una, sino muchas modalidades de paz. Dichas formas comprometen el orden de lo preestablecido y buscan dar un nuevo orden. Acá, la respuesta que se dará inicialmente es: la paz es para los vencidos... Creo 
que ese es el primer reconocimiento, no creo necesaria la adopción del término de Walter Benjamin como categoría universal, pero sí como un primer momento de la historia por construir, a saber, la historia de la paz. A lo largo del texto podemos ver cómo este término ("vencidos") se puede transfigurar en la expresión actores de paz; entonces, para una pregunta intermedia como ¿cuándo se deviene actor de paz?, la respuesta es la tarea a elaborar aquí.

Como se verá, al ir atendiendo a estas preguntas y al ir comprometiéndonos cada vez más con ellas, nos daremos cuenta de que las diversas formas de hacer la paz, en presente, y los retos y desafíos por asumir, en futuro, dan cuenta de una paz difícil, en cuanto exigente. Una nueva forma de vida - más de las veces, dura de asir, en la medida en que implica tratar de darle la vuelta a maneras de ser y de hacer históricas - implica, como diría Benjamín, “cepillar el curso de la historia a contrapelo" (Tesis IV). ${ }^{1}$

En este sentido, el trabajo se desarrolla en dos momentos: en el primero, se habla acerca de quiénes son/somos los vencidos y cómo devienen actores de paz; en el segundo, se expone esta idea de la paz difícil, en el marco de los procesos y diversas configuraciones de hacer las paces ${ }^{2}$.

\section{De los vencidos a los actores de paz}

Walter Benjamin en sus Tesis sobre el concepto de historia (2005), al tiempo que está preocupado por el concepto de historia, está preocupado por la situación tensionante entre dominadores y dominados, opresores y oprimidos, vencedores y vencidos. Porque, para él -y todas las

1 En adelante se citarán de esta manera los apartados de las tesis de Walter Benjamin, para que el lector pueda consultarlos más fácilmente en cualquier edición impresa o virtual.

2 Uno de los esfuerzos más grandes en relación con la construcción del presente texto consiste en superar toda inmediatez informativa, para poder pensar y proponer un orden que vaya mucho más allá de la coyuntura, aunque la asuma per se. En los últimos años, ha sido tanta y tan basta la producción en torno a la paz, que se tiende a pontificar sobre ella, sin ningún tipo de fundamento o, lo que es peor, sin ningún tipo de postura crítica. 
formas de pensar históricamente en el XIX le respaldan-, la historia que sabemos y contamos es la historia de los vencedores, jamás la de los vencidos. Por debajo de esta concepción de la historia descansa la inevitable y peligrosa idea del progreso. Esa misma idea que ha derramado sangre durante años y que justifica, por ejemplo, que para tener paz es necesario hacer la guerra, lo que en algunas teorías se ha entendido como la paz romana (Galtung, 1996, 45). Dice Benjamin en un célebre pasaje: "no existe documento de cultura que no sea a su vez documento de barbarie" (Tesis III).

Dentro de las cuestiones que analiza el filósofo, está inmersa la pregunta por quiénes son los vencidos. A continuación, a expensas de la idea originaria de Benjamin, sugiero las características de tres tipos de vencidos, en clave de las intenciones particulares de este texto ${ }^{3}$ :

1) Aquellos para quienes solamente hay una manera de contar la historia: la historia de la guerra, la historia de los vencedores, la historia del progreso.

2) Aquellos que restan indiferentes ante los discursos de aparente desarrollo que detentan desigualdad.

3) En la línea de los dos anteriores, aquellos que suspenden la propia conciencia histórica y la propia condición política en el curso del tiempo. En otras palabras, no hay mayor vencido que aquel que renuncia a su condición política, en tanto que sujeto de la historia.

Cuando se abordan estas tres formas de comprensión acerca de quiénes son los vencidos en sentido amplio, entendemos entonces que cada una nos da la llave de un tema por superar. Cada forma de ser vencido

3 Como se puede evidenciar, al hablar de "vencidos" el presente texto no tiene ni una vocación militar - puesto que no se refiere necesariamente a aquellos que pierden un combate armado- ni expone una relación directa entre vencidos y víctimas, aunque ciertamente sí puede haber una relación. En otras palabras, las víctimas no son necesariamente los vencidos, como se verá. 
nos pone delante de un asunto por resolver como grupo social, como comunidad política.

El primer tipo de vencido implica la resolución de la forma en que asumimos la historia y cómo nos asumimos en ella. Hay una caricatura en la que se aprovecha la vieja frase "el que no estudia su historia está condenado a repetirla", para añadir: "y el que la estudia está condenado a verla repetirse por culpa de los que no la estudian". Recordemos que nadie puede reconocer la historia, sin reconocerse al mismo tiempo en ella. "El sujeto del conocimiento histórico es la misma clase oprimida que combate” (Tesis XII).

Figura 1. "Quienes no conocen la historia están condenados a repetirla"

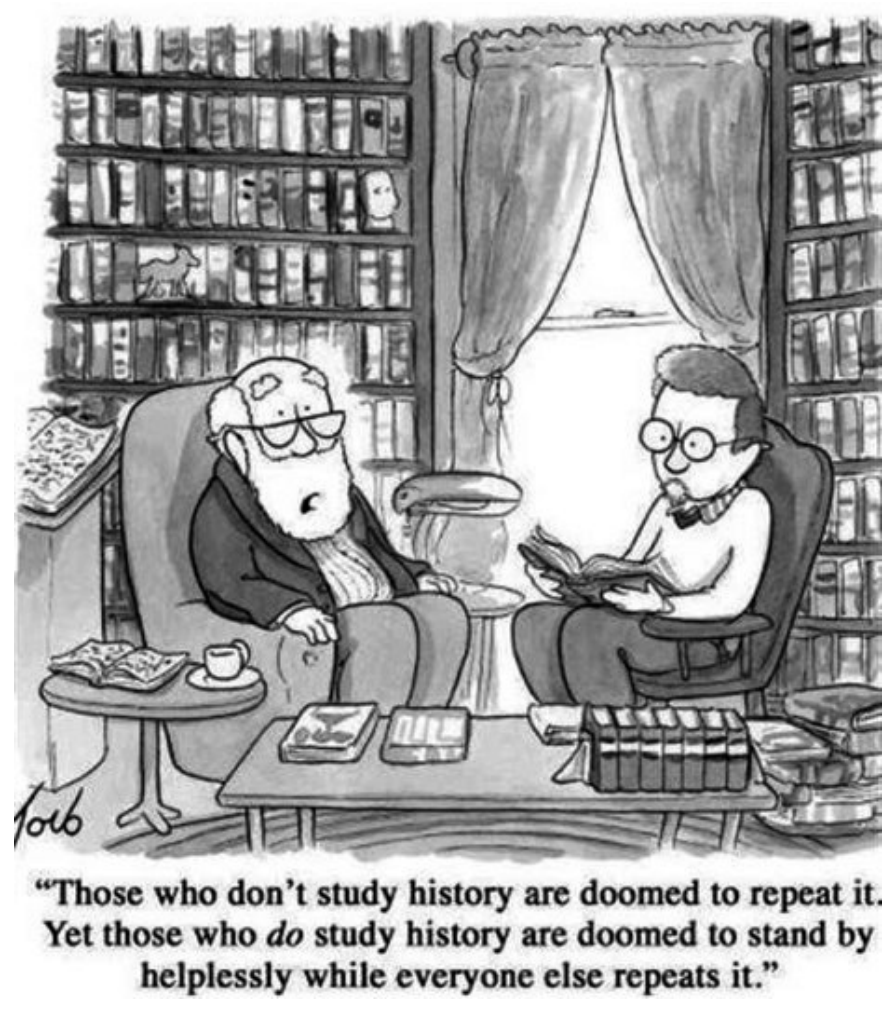

Fuente: Tom Toro. 
Día a día, nuestras acciones respaldan una historia que se cuenta a favor de unas clases sociales, en detrimento de otras. Esto, de hecho, es lo que ha detonado la lucha armada de millones de grupos en el mundo, ya que los vencidos están siempre llamados a superar su condición como tales. Aunque muchas veces se hay interpretado que el camino a seguir debía ser exclusivamente el de la guerra de los vengadores, también hay formas de hacer lucha política en la paz ${ }^{4}$, de poner a pulso voluntades, de mostrar que las paces se hacen con los enemigos, pero también con los amigos. Solo que ni una lucha ni otra (ni la armada ni la desarmada) pueden ser ahistóricas. Para el caso concreto, son muchos los que quieren escribir la historia de la paz, pero no saben cómo, de modo tal que, frente a la pregunta ipara quién es la paz?, diríamos: para aquel que se asuma a sí mismo como sujeto de la historia, ya que "la historia es objeto de una construcción cuyo lugar no es el tiempo homogéneo y vacío, sino el “tiempo actual”” (Tesis XIV).

En el segundo tipo de vencido, se observa la ausencia de comprensión, en la que disociamos que todo proyecto político, además de histórico, es un proyecto económico, que toca tanto intereses privados como públicos. En esta línea, así como la lucha política de la paz no es apolítica, mucho menos es aeconómica. Anunciar una voluntad de paz toca puntos sensibles, entre ellos, la posibilidad de imaginar un mundo que suspenda las violencias mismas del capitalismo en el proyecto económico neoliberal; esto supone la comprensión de que, así como hay una economía de la guerra, también hay una economía de la paz. En esta medida, aunque el principio de la paz sea la vida, su objetivo perfectible es el viejo problema de cómo se debe vivir. Junto al principio de la vida, está el de "la paz con justicia social"s. La lucha por la paz no

4 Si bien es cierto que la reflexión desde el marxismo crítico ha hecho devenir vencidos en vengadores, como dice Benjamin en la tesis XII, ello no significa que no pueda alimentar también la transformación de los vencidos en actores de paz, con lo que daría un giro de $180^{\circ}$, para pasar de discursos verticales a discursos horizontales que, sin suspender la lucha política, desarrollen el principio de la no violencia activa.

5 Aquel en el que tanto insistía Carlos Pizarro en sus más variados escritos y discursos. 
tolera una economía neoliberal, pues esto amenaza de nuevo con "convertirnos en instrumento de la clase dominante" (Tesis VI).

En ocasiones se suele confundir al sujeto de paz con el sujeto de la tolerancia, capaz de soportar todo con tal de evitar una guerra. Esta incomprensión descansa en pensar la paz al margen de la lucha política en paz, que es distinto. En la vía negativa, la paz no es tolerar con abnegación, la paz es inscribirse y llevar la lucha política en contra de una de las raíces más profundas de las distintas formas de violencia: el proyecto económico capitalista y su ethos neoliberal ${ }^{6}$, en el que, para garantizar sus condiciones de vida, unos explotan tanto a la naturaleza como a los trabajadores: "tal concepción no quiere ver más que los progresos del dominio sobre la naturaleza y se desentiende de los retrocesos de la sociedad" (Tesis XI). De aquí que, todo proyecto de paz, a la vez que apuesta por el cuidado de la naturaleza, toca también las condiciones de trabajo, con lo cual, aparecen nuevamente los enemigos y los contradictores de una paz que se busca en la lucha política.

En relación con el tercer y último tipo de vencido, como síntesis de la exigencia de los dos anteriores, encontramos la comprensión de que no puede haber paz sin sujeto político, que asuma un compromiso con la historia y se asuma como parte de ella. Así, frente a la pregunta ipara quién es la paz?, diríamos: para todo aquel que comprenda que, como construcción social, no puede haber paz sin sujeto político (Tabares, 2010 , p. 6). El mayor vencido de la historia es el hombre y la mujer que se separan de su condición política, puesto que con esta dimisión naturalizan su historia, se adhieren al proyecto hegemónico de desarrollo

6 Un chiste que circula por las redes sociales, cuya función, además de hacer reír, consiste en promover el uso de la bicicleta dice: “ ¿Es gordo y feo? Sea solo feo, compre una bicicleta”. Algo parecido podría hacerse con ciertos partidos políticos que eligen todavía la guerra por sobre la paz: “ ¿Es usted violento y corrupto? Sea solo corrupto, diga sí a la paz”. Lo cual estaría bien para un primer momento; pero, así como no es posible la paz y al mismo tiempo la solución de los conflictos por la vía armada, tampoco es posible la paz y las formas de gobierno corruptas (o no por lo menos en las medidas de corrupción de la Colombia actual). 
y renuncian de manera definitiva a la construcción de la paz? . De aquí que se pueda superar la condición de vencido y asumir el papel de actor de paz, de paces, al que hoy nos convoca nuestro tiempo.

\section{La paz difícil y los actores de paz}

El anterior apartado nos puede hacer caer en la tentación de considerar que el sujeto político es solo aquel sujeto cuya formación social, política, económica e histórica, le permite un panorama completo o, como suele decirse, una visión holística de todo un estado de cosas en su actualidad. Pero no. No hace falta todo ello para devenir actor de paz. Como ejemplo para alcanzar mayor claridad, en el marco de los ejercicios de reconciliación con la comunidad no-víctima, que practican miembros de la Fundación Ágape por Colombia ${ }^{8}$, aparecen frente a un tablero dos mujeres: una a la derecha y otra a la izquierda. Ambas comienzan a dibujar: la de la izquierda dibuja tres ramas secas, la una sobre la otra, en un espacio vertical; la de la derecha, dibuja una semilla, y debajo un árbol que comienza a crecer, bajo el cual se observa un árbol con frutos florecidos. Luego, miran fijamente al público y la de la izquierda dice primero: "yo era víctima, sigo siendo víctima”; a su vez, la de la derecha dice: "yo era víctima, ahora soy actor de paz".

En este punto se evidencia un tipo de conocimiento sobre lo social, sobre los procesos de reconciliación consigo mismo y con una comunidad específica, que no es necesariamente académico, pero que puede ir incluso más allá del mismo en el marco de las luchas y las organizaciones sociales, que tienen y aportan sus propios conocimientos para la

7 Ciertamente, esta idea no está en Benjamin. Acá aprovecho el recurso para actualizarlo y potenciarlo en las formas de vida actuales.

8 Esta fundación tiene cerca de catorce años de experiencia en el trabajo con víctimas, victimarios, jóvenes desvinculados y adultos desmovilizados, a través de ejercicios de reconciliación. Es posible decir que, en estos conocimientos está el saber del perdón, del respeto por el otro, de la capacidad de una comunicación asertiva, entre otros. 
consecución de la paz. Dice, por ejemplo, un líder de la comunidad wuayú: "somos una comunidad que ha desarrollado el diálogo [...]. Estamos aquí diciéndole al país que se puede vivir desde las diferencias sin violencia, usando el poder de la palabra" (Hernández, 2013, p. 46). Existe toda una bibliografía reciente con innumerables ejemplos de grupos conformados por comunidades indígenas, afro y campesinas, así como por movimientos ciudadanos de paz, que son una muestra de ello y que corroboran las tesis propuestas hasta ahora?

En la tradición marxista, oprimidos, dominados o subalternos están llamados a hacer lucha política abierta y, como se dijo anteriormente, sobre todo, desde la vía violenta. Según Guevara, "el último recurso" se hizo el primero en la agenda política para resolver conflictos. Se trata de una cuestión que lleva muchos años perpetuándose y que se ha tramitado sin tener en cuenta lo que, por ejemplo, muchas culturas indígenas reconocen como el poder de la palabra. Así, cuando aparecen viejas formas para tramitar los conflictos, e incluso obvias, es menester recordar de quiénes pueden venir estas iniciativas. En el tiempo presente, solemos pensar que han venido solamente de un grupo conformado por el Estado, encabezado por Juan Manuel Santos, y el grupo guerrillero más antiguo de Latinoamérica, las FARC-EP. Con ello, solemos descuidar el hecho de que hay muchos otros grupos sociales, colectivos, organizaciones, asociaciones, que se han conformado históricamente, desde mucho antes del 12 de noviembre de 2014, y cuya pregunta por la historia no es solo por quiénes somos o fuimos, sino por cómo nos reinventamos como sociedad.

9 Los ejemplos en esta línea son innumerables y ya hay una buena cantidad de bibliografía sobre las experiencias de paz. Recomiendo, en esta línea, algunos trabajos como el libro de la profesora Esperanza Hernández, Intervenir antes que anochezca (2012); el libro de la profesora Esperanza Gómez Hernández, Diálogo de saberes e interculturalidad (2015), que desarrolla a profundidad la compleja categoría de "diálogo de saberes"; y el trabajo de los profesores Luis Alberto Arias y Patricia Carrera, Las organizaciones indígenas y afrodescendientes desplazadas en Bogotá y sus resignificaciones culturales (2016). 
Muchos grupos sociales como los anteriormente mencionados han adelantado múltiples esfuerzos por reescribir la historia de la guerra y transfigurarla en nuevas formas de paz. Aparecen sin tener en cuenta ningún tipo de teoría intelectual y así contribuyen a construir formas de vida distintas a cualquier violencia directa, estructural o cultural (Galtung, 1985). La importancia, como se decía al inicio, radica en una sana comprensión de lo plural en lo que refiere a las construcciones de paz. En un extraño fenómeno gramatical, podríamos llegar a ver que una pluralidad de acciones, como hacer las paces de formas diversas y múltiples, tenga como consecuencia La Paz con la que soñamos, en singular.

De este modo, se puede decir que una buena parte de estos grupos, colectivos y organizaciones, poseen, dentro de sus conocimientos, una conciencia histórica que les permite construir la paz desde sus maneras de vivir y, en ello, comprenderse como sujetos. Desde allí, dejan grandes lecciones acerca de cómo la sociedad tiene la capacidad de vivir en paz, de vivir construyendo la paz. De hecho, este conocimiento sobre la paz radica en gran medida en la comprensión de dos cosas: una, que la paz realizable no es precisamente una paz perfecta, como la que explica el profesor Francisco Muñoz en su texto La paz imperfecta (2000); y dos, que, para el caso colombiano, no solamente es imperfecta, sino además difícil, tanto en sus fines como en sus medios. En primer lugar, es difícil, porque compromete, como diría Rancière, el orden de lo prestablecido (2004). Asimismo, pensemos - y por cuestiones de espacio, de forma superficial- en las diferentes maneras en que compromete el orden de la actividad política, el orden de lo económico, el orden de lo mediático, el orden de lo educativo y, por supuesto, el orden de lo cultural ${ }^{10}$.

10 Soy consciente de que cada uno de estos elementos conllevaría un análisis y una serie de reflexiones mucho más detalladas de las que aparecen acá. El objetivo en este punto es generar el deseo del lector por profundizar en cada uno de los aspectos, si se quiere, apoyado por la suma bibliográfica que ha venido apareciendo y que se compila de forma ordenada y completa al final de texto. 
La paz difícil implica estar preparados para la manipulación de sus condiciones a nivel estatal, mediático, empresarial y educativo. La manipulación de la paz no es otra cosa que la negación o la oposición que hay de parte de las formas del poder en contra de la autonomía que ganan las distintas maneras de organización social. A nivel político, tal y como nos lo recuerda la Comisión Histórica del Conflicto y sus Víctimas en los distintos ensayos del texto Contribución al entendimiento del conflicto armado en Colombia (2015), la violencia en este país se ha alimentado por los cierres en el universo político (de Zubiría, p. 134), desde las formas tradicionales de hacer política con el bipartidismo, hasta las formas actuales, que temen e infunden temor sobre cualquier proyecto distinto de los acostumbrados, para generar, desde la persecución política física y simbólica, la no aceptación de lo distinto y, con ello, de las formas originales y autónomas de construir paz ${ }^{11}$.

En este sentido, las paces tienen que coincidir con una serie de aperturas del universo político, las cuales comienzan justamente por el autorreconocimiento que debe hacer el indiferente, o el llamado sujeto despolitizado, de su papel en la historia. Este ha de extenderse a la aceptación de otras formas de configurar sociedades, más allá de las conocidas, en las que todos y cada uno de los movimientos sociales ${ }^{12}$ juegan un papel importante. Otra distinción importante es que, así como la guerra se juega dentro y fuera de una economía, también es necesario comprender las economías de la paz, entender la paz en las lógicas de un mercado, para no cometer el error de sacralizar lo profano que hay en la paz o de profanar lo sacro que hay en las formas como se

11 No hace falta recordar que, mientras hacemos apuestas concretas por la paz, en lo recorrido entre el 2016 y el 2018 han sido asesinados alrededor de 217 líderes sociales. Para mayor información, véase: https://goo.gl/aCSNKJ

12 Para profundizar en el estudio de este punto, la lectura del ensayo de Sergio de Zubiría, que aparece en el texto citado, Contribución al entendimiento del conflicto armado en Colombia (2015), resulta un apoyo fundamental. Así mismo, encuentro importante el informe del Centro de Memoria, Paz y Reconciliación, Debates de la memoria-aportes de las organizaciones víctimas a una política de la memoria (2014). 
construye paz. Con este objetivo, cabe pensar de nuevo en las ideas del mismo Benjamin acerca de lo sagrado y lo profano, desarrolladas para una filosofía política del tiempo presente por el filósofo italiano G. Agamben, en su libro Profanazioni (2005).

Los actores de paz comprenden que una apertura de lo político es también una apertura de cómo entendemos la economía y qué entendemos por desarrollo ${ }^{13}$; no solo las formas de vida de la guerra se oponen a la paz, sino también las formas de vida cuyo mercado se basa en la explotación ambiental y del trabajo. Darle la vuelta a esto, supone el trabajo juicioso de examinar otro tipo de sustento y autosustento, implica lo que muchos han entendido como hacer las paces con la tierra y hacer las paces con el trabajo mismo ${ }^{14}$. Esta cuestión deja ver que la paz no solo es el problema social de unas cuantas naciones con conflictos internos, sino un problema de carácter global, aun cuando no en todas partes del mundo haya guerra.

En lo que refiere a la cuestión sobre el papel de lo mediático, mientras no se atienda al hecho de que la opinión pública está condicionada por lo que producen y reproducen los medios masivos de comunicación, quienes, en términos particulares, obedecen a los intereses de las clases

13 Acá podemos hacer una distinción epistemológica, de consecuencias políticas. Una vieja distinción que todavía no es lo suficientemente clara y que por ello vale la pena seguir insistiendo en ella. Decimos, entonces, que no todo desarrollo implica progreso ni todo progreso implica desarrollo. Históricamente, hemos sido testigos de cómo proyectos económicos, en nombre del progreso, se oponen al desarrollo humano y al acontecer mismo del mundo natural, puesto que se destruye de las más variadas formas. Por el contrario, si entendemos el desarrollo humano como formas de vida capaces de convivir en paz con el medio ambiente, estaremos, ahora sí, hablando de progreso - si se quiere, de algo así como un "posprogreso"-. En cualquier caso, ambos conceptos son difíciles porque son usados como sinónimos e indistintamente.

14 Sobre este punto, enumero los siguientes trabajos: el artículo de la profesora Hernández "Resistencias para la paz en Colombia: significados, expresiones alcances” (2009), "Replanteando la epistemología de la paz: el caso de la decolonización de la paz" de Victoria Fontan (2012) y "La paz imaginada por quienes la construyen: iniciativas civiles de paz de Base Social”, de Esperanza Hernández (2008). 
dominantes, será técnicamente imposible lograr la paz. Recuerdo que, en una conferencia, pocos días antes del plebiscito, me preguntaron por la posibilidad de la victoria del "no", desde la propaganda política hecha por un canal como RCN. Era corto el tiempo de respuesta y solo acerté a apelar a un argumento de autoridad: "un país que no le cree a sus víctimas y a sus intelectuales, no merece la paz". El papel de los medios es fundamental, no solo en términos de decisiones políticas, como lo era en este caso del plebiscito, sino por lo que significa para una buena cantidad de consumidores en términos de representación e imaginarios. Por ejemplo, cuando un titular presenta la muerte de un guerrillero a manos de la fuerza pública como un acto heroico y, en el caso contrario, como un acto terrorista, hace evidente, para quien es capaz de verla, la manipulación de la información; asimismo, a quien no es capaz de verla, le da una formación ideológica muy precisa y un mensaje dogmático claro.

Otro aspecto importante es el educativo. Toda paz es, cuanto menos educada, cuanto más difícil. Y esto quiere decir varias cosas. La primera de ellas es plantearse la pregunta y la respuesta del texto La paz en la educación, cuando dice:

¿debe esperarse a tener una sociedad en paz para educar para la paz, o es posible educar para la paz en medio de la cultura de guerra y de las guerras vigentes y existentes? Ambigüedad que pone a pensar en una educación real a la paz, ya que de hecho hay que educar a la paz en una sociedad que no está en paz, no solo porque existe la guerra, sino porque los valores que priman son los de la guerra (Jiménez, 2014, p. 3).

La segunda es decir que, así como cada uno de los puntos anteriores implica un momento crítico que evidencia un aspecto tensionante, como para el caso de lo que se tiene y lo que está por hacerse a nivel político, económico y mediático, así mismo en la cuestión educativa, y es que, solamente una educación en hacer crítica, como comprender los momentos anteriores, es la garantía de asumir la paz con todo y sus 
dificultades. Ya que comprender los momentos anteriores es evidenciarnos a nosotros mismos en los pros y los contras de nuestras formas de vida más íntimas y cómo en el marco de las mismas, construimos, exigimos y asumimos la paz.

\section{Conclusiones}

En el film X-Men Primera Generación aparece la amenaza de una guerra inminente: si uno de los dos bandos (estadounidenses o rusos) cruza una línea marítima invisible, será el comienzo de la guerra. Esa línea invisible es la de la línea de la paz, que, además de invisible, es frágil, pues se construye con hilos delgados y finos, que pueden llegar a llamarse fuertes y sólidos, solamente si se logra la unión de aquellos a quienes hemos llamado "los vencidos", para llamarlos en adelante, y con justicia, actores de paz.

No hace falta esperar la paz para devenir sujetos políticos; esta es una oportunidad para asumirnos como tales, para asumir esa paz difícil y comprometernos con ella siempre en plural. En razón a esto, es importante configurar y hacer parte de los distintos movimientos sociales que de forma crítica le apuestan a la paz; así, al asumir la paz como difícil, asumimos con ella la capacidad de hacer saltar el "continuum de la historia” (Tesis X). De modo que, la siguiente pregunta a responder tiene un profundo sentido epistemológico: ¿qué saberes ponemos en juego en nombre de un interés común como lo es la paz?

Para citar el asunto de la pequeña correspondencia entre Einstein y Freud, que se conoce bajo el título “¿Por qué la guerra?”, la respuesta, creo, podría ser, a cambio, para qué la paz, puesto que allí, entre líneas, aparece la paz como forma de gobierno y de autogobierno: la paz como la mejor forma de hacer política, la paz como el paso complejísimo del derecho del más fuerte (el derecho de los iguales) a la igualdad de derechos. En este sentido, el espacio de cultura de paz no ha de verse como una pacificación homogénea, sino como un espacio de lucha política, el 
cual no solo no contempla la violencia, sino que fomenta los principios de la vida social en común. Esto exige claramente pasar de los cierres políticos, epistemológicos, económicos y mediáticos, a una verdadera apertura a la democracia.

Por último, no está de más decir que este texto no se presenta tanto como una versión de cómo debería hacerse la historia, sino de cómo debería seguirse haciendo, en la medida en que reconoce que, más allá de lo que suele ser visto y visibilizado, existe una cantidad innumerable de personas y grupos comprometidos con hacer las paces, la paz.

\section{Referencias}

Acodesi (2003). Hacia una educación para la paz. Bogotá: Kimpres.

Agamben, Giorgio (1970). El hombre sin contenido. Barcelona: Altera.

Arias, Luis Alberto y Carrera, Patricia (2016). Las organizaciones indígenas y afrodescendientes desplazadas en Bogotá y sus resignificaciones culturales. Bogotá: Fundación Universitaria Unimonserrate.

Benjamin, Walter (2005). Tesis sobre una filosofía de la historia. (Echeverría Bolívar, trad.) Recuperado de: https://goo.gl/Mdzpqh

Bloomfield, David (2003). Recontiliation after violent confict. Stockholm : International Institute for Democracy and Electoral Assistance.

Cabezudo, Alicia (2012). Educación para la paz: una construcción de la memoria, la verdad y la justicia. Desafío pedagógico de nuestro tiempo en América Latina. Ciencias Sociales Unisinos, (48), 139-145.

Cerdas, Evelyn (2015). Desafíos de la educación para la paz, hacia la construcción de una cultura de paz. Revista Electrónica Educare, 2 (19), 135-154. Recuperado de: https://goo.gl/Y29Lwj

Derrida, Jacques (2003). El siglo y el perdón. Buenos Aires: Ediciones La Flor.

Dueñas, Manuel y Rodríguez, María (2002). Educar para la paz enseñando historia. Investigación \& Desarrollo, 10 (1), 40-53. Recuperado de: https:// goo.gl/zpJX3r 
Espitia, Carlos (2015). En la ruta hacia la paz. Debates hacia el fin del conflicto y la paz duradera [serie de ensayos, resultado del diplomado La paz es ahora]. Bogotá: Centro de Memoria Histórica.

Fernández, Alfonso y López, María (2014). Educar para la paz. Necesidad de un cambio epistemológico. Convergencia. Revista de Ciencias Sociales, 21 (64), 117-142. Recuperado de: https://goo.gl/F2fXz8

Freire, Paulo (1970). Pedagogía del oprimido. México: Siglo XXI.

Freire, Paulo (2002). Cartas a quien pretende enseñar. México: Siglo XXI.

Freire, Paulo (1996). Política y educación. México: Siglo XXI.

Galtung, Johan (1985). Sobre la paz. Barcelona: Fontamara.

Gaultung, Johan (1996). Peace by paceful means. Peace and conflicts, development and civilization. Oslo: Prio.

Gómez, Esperanza (2015). Diálogo de saberes e interculturalidad. Medellín: Pulso y Letra.

Gramsci, Antonio (1997). Quaderni del carcere, VI. Torino: Eniaudi.

Hayner, Priscilla (2011). Unspeakable truths-transitional justice and the challenge of truth commissions. New York: Routegle.

Hernández, Esperanza (2009). Paces desde abajo en Colombia. Reflexión Política, 11 (22), 176-186. Recuperado de: https://goo.gl/kCdK1u

Hernández, Esperanza (2012). Intervenir antes que anochezca. Bucaramanga: Universidad Autónoma de Bucaramanga.

Horkheimer, Max (1937). Teoría tradicional y teoría crítica. Barcelona: Paidós.

Jiménez, Francisco (2014). Una historia de la investigación para la paz. Historia Actual Online, 34, 142-162. Recuperado de: https://goo.gl/2V3dKH

Kant, Immanuel (2016). La paz perpetua. España: Alianza Editorial.

Matyòk, Thomas, Senehi, Jessica y Byrne, Sean (2011). Critical issues in peace and conflict studies. New York: Lexington Books.

Molano, Alfredo. (2011). Fragmentos de la Historia del conflicto Armado (19202010). Colombia: Espacio Critico. 
Muñoz, Francisco. (2005). La paz imperfecta ante un universo en conflicto. Instituto de la Paz y los Conflictos de la Universidad de Granada. Recuperado de: https://goo.gl/ZS2Yv2

Comisión Histórica del Conflicto y sus Víctimas (2016). Contribución al entendimiento del conflicto armado en Colombia. Comisión histórica del conflicto y sus víctimas. Bogotá: Desde Abajo.

Rancière, Jacques (2010). La noche de los proletarios: archivos del sueño obrero. Buenos Aires: Tinta limón.

Reyes, Manuel (1991). La razón de los vencidos. Madrid: Antrophos.

Hernández, Luis y García, Yadira (2014). Víctimas, victimarios: El papel de las universidades en la construcción de cátedras de paz. Jurídicas CUC, 10 (1), 27-42. Recuperado de: https://goo.gl/8WSABR

Martínez, Vicent, Comins y Irene, París, Sonia (2009). La nueva agenda para la filosofía del siglo xxi: los estudios para la paz. Convergencia, 91-114. México: UNAM.

Martínez, Vicent; Comins, Irene y París, Sonia (2009). La nueva agenda de la filosofía para el siglo xxI: los estudios para la paz. Convergencia. Revista de Ciencias Sociales, (16), 91-114.

Martínez, Vicent (2000). Saber hacer las paces: epistemología de los estudios para la paz. Convergencia. Revista de Ciencias Sociales, 7 (23), 49-96. Recuperado en https://goo.gl/64Lx9k

Massó, Ester (2007). El desafío de la paz como quehacer humano: Retos [antropológicos, sociales, políticos] de culturas y pueblos. Derechos humanos, cultura (s) de paz (primera parte). Limite. Revista Interdisciplinaria de Filosofía y Psicología, 2 (16), 25-45. Recuperado de: https://goo.gl/zLHhjz

Nasi, Carlo y Rettberg, Angelika (2005). Los estudios sobre conflicto armado y paz: un campo en evolución permanente. Colombia Internacional, (62), 64-85. Recuperado de: https://goo.gl/psPPJS

Villar, María y Maldonado, Ana (2013). Los medios de comunicación y su injerencia en la construcción de cultura de paz o violencia. Una reflexión desde los estudios para la paz. Ra Ximhai, 9 (3), 47-63. Recuperado de: https://goo.gl/X2rP1R 